EFDA-JET-CP(05)02-45

J.P. Graves, C. Angioni, R.V. Budny, R.J. Buttery, S. Coda, L.-G. Eriksson, C.G. Gimblett, T.P. Goodman, R.J. Hastie, M.A. Henderson, H.R. Koslowski, M.J. Mantsinen, An. Martynov, M.-L. Mayoral, A. Mück, M.F.F. Nave,

O. Sauter, E. Westerhof and JET EFDA contributors

\title{
Sawtooth Control in Fusion Plasmas
}





\section{Sawtooth Control in Fusion Plasmas}

J.P. Graves ${ }^{1}$, C. Angioni ${ }^{2}$, R.V. Budny ${ }^{3}$, R.J. Buttery ${ }^{4}$, S. Coda ${ }^{1}$, L.-G. Eriksson ${ }^{5}$, C.G. Gimblett ${ }^{4}$, T.P. Goodman ${ }^{1}$, R.J. Hastie ${ }^{4}$, M.A. Henderson ${ }^{1}$, H.R. Koslowski ${ }^{6}$, M.J. Mantsinen ${ }^{7}$, An. Martynov ${ }^{1}$, M.-L. Mayoral ${ }^{4}$, A. Mück ${ }^{1}$, M.F.F. Nave ${ }^{8}$, O. Sauter ${ }^{1}$, E. Westerhof ${ }^{9}$ and JET EFDA contributors*

${ }^{1}$ Centre de Recherches en Physique des Plasmas, Association EURATOM-Conf'ed'eration Suisse, EPFL, 1015 Lausanne, Switzerland

${ }^{2}$ MPI f ur Plasmaphysik, EURATOM-Association, D-85748 Garching, Germany

${ }^{3}$ Princeton Plasma Physics Laboratory, Princeton, NJ, 08543, USA

${ }^{4}$ UKAEA/Euratom Fusion Association, Culham Science Centre, Abingdon, UK

${ }^{5}$ Association EURATOM-CEA, CEA-Cadarache, F-13108 St. Paul lez Durance, France

${ }^{6}$ Association EURATOM-FZ-Juelich, Institut fuer Plasmaphysik, Trilateral Euregio Cluster, D- 52425 Juelich, Germany

${ }^{7}$ Association Euratom-Tekes, Helsinki University of Technology, Finland

${ }^{8}$ Associa $_{s}$ cao EURATOM/IST, Centro de Fus ao Nuclear, Lisbon, Portugal

${ }^{9}$ FOM-Institute for Plasma Physics Rijnhuizen, Association EURATOM-FOM, Trilateral Euregio Cluster,

The Netherlands

* See annex of J. Pamela et al, "Overview of JET Results",

(Proc. $20^{\text {th }}$ IAEA Fusion Energy Conference, Vilamoura, Portugal (2004).

Preprint of Paper to be submitted for publication in Proceedings of the

EPS Conference,

(Tarragona, Spain 27th June - 1st July 2005) 
"This document is intended for publication in the open literature. It is made available on the understanding that it may not be further circulated and extracts or references may not be published prior to publication of the original when applicable, or without the consent of the Publications Officer, EFDA, Culham Science Centre, Abingdon, Oxon, OX14 3DB, UK."

"Enquiries about Copyright and reproduction should be addressed to the Publications Officer, EFDA, Culham Science Centre, Abingdon, Oxon, OX14 3DB, UK." 


\begin{abstract}
Clear observations of early triggering of neo-classical tearing modes by sawteeth with long quiescent periods have motivated recent efforts to control, and in particular destabilise, sawteeth. One successful approach explored in TCV utilises electron cyclotron heating in order to locally increase the current penetration time in the core. The latter is also achieved in various machines by depositing electron cyclotron current drive or Ion Cyclotron Current Drive (ICCD) close to the $q=1$ rational surface. Crucially localised current drive also succeeds in destabilising sawteeth which are otherwise stabilised by a coexisting population of energetic trapped ions in the core. In addition, a recent reversed toroidal field campaign at JET demonstrates that counter-Neutral Beam Injection (NBI) results in shorter sawtooth periods than in the Ohmic regime. The clear dependence of the sawtooth period on the NBI heating power and the direction of injection also manifests itself in terms of the toroidal plasma rotation, which consequently requires consideration in the theoretical interpretation of the experiments. Another feature of NBI, expected to be especially evident in the negative Ion based Neutral Beam Injection (NNBI) heating planned for ITER, is the parallel velocity asymmetry of the fast ion population. It is predicted that a finite orbit effect of asymmetrically distributed circulating ions could strongly modify sawtooth stability. Furthermore, NNBI driven current with nonmonotonic profile could significantly slow down the evolution of the safety factor in the core, thereby delaying sawteeth.
\end{abstract}

\title{
1. INTRODUCTION
}

The control of sawteeth is expected to be important in a next step device such as ITER [1]. The presence of highly energetic ions in large tokamaks has given rise to sawteeth with long quiescent times and large amplitudes [2] -[4]. Although this might seem to be an advance, large sawteeth also have detrimental ramifications. In particular, the radial location of the collapse event propagates with respect to the sawtooth quiescent time. The collapse radius has been predicted to be so large in ITER [5] that coupling is likely to occur with modes at other rational surfaces. Evidence of interaction between large sawteeth and 3/2 Neoclassical Tearing Modes (NTM) has been observed in JET [6], while discharges with smaller regular sawteeth are found to have increased core confinement, and are less likely to be coupled to confinement degrading NTMs[6]. Hence it is seen that greater understanding and control over the mechanisms that determine sawteeth stability is required.

Sawtooth control refers to the ability of an actuator system, i.e. some additional heating and/or current drive, to alter the period and usually the amplitude of the sawtooth instability. Two strategies are possible to avoid the deleterious effects of large sawteeth. One option which has had some success in JET [7] is to attempt to suppress sawteeth for many energy confinement times. In ITER one would attempt to reach burning conditions before the onset of the first crash. This may prove possible because of the expected stabilising properties of the trapped alpha population [8]. Moreover, as we shall discuss later, co-injection of negative ion based neutral beams in ITER's baseline scenario could suppress the formation of a $\mathrm{q}=1$ rational surface over a significant fraction of the current penetration timescale. The other strategy is to deliberately increase the rate of sawteeth, i.e. destabilise 
sawteeth, in order to reduce the perturbation to the plasma beyond the inversion radius. The most successful and repeatable experiments involve Radio Frequency (RF) heating and current drive with the resonant surface localised close to the $q=1$ rational surface. Evidently the control mechanism involves the destabilising effect of creating local perturbations in the current and electron temperature profiles $[9,10]$. Another approach involves the injection of neutral beams with orientation opposite to the direction of the plasma current [11]. Such experiments point to the possible sensitivity of sawteeth to momentum induced plasma rotation.

This paper collects recent experiments and simulations which employ localised Electron Cyclotron Heating $(\mathrm{ECH})$ and Current Drive (ECCD) $[10,12,13]$ and Ion Cyclotron Resonance Heating (ICRH) and Current Drive (ICCD) $[6,12,14]$ to control sawteeth. The reproducibility of such experiments has recently been exploited in order to destabilise sawteeth by localised ICCD, and thereby control sawteeth which were initially lengthened by a population of energetic ions in the core [15]. This has important and encouraging implications for the control of sawteeth in a next step tokamak, since the experiments provide evidence that local modification of the current profile could assist destabilisation of sawteeth despite the predicted large energy sink $\delta \mathrm{W}$ from the kinetic contribution of trapped alpha particles in the core. Also presented in this paper are dedicated experiments and analysis of counter-injection (counter in relation to the plasma current and toroidal field) of neutral beams in JET [16]. It will be seen that the observation of sawteeth in discharges employing moderate counter-NBI, with quiescent times not exceeding those of Ohmic discharges, can be partially explained in terms of a reduction in stabilising kinetic contributions to $\delta \mathrm{W}$. Finally, recent experimental [17] and theoretical [18] results on negative ion based neutral beam injection (NNBI) are reviewed. Despite NNBI populations having a dominant passing fraction, the sawteeth are strongly stabilised by the energetic population. It is shown in this article under what conditions the NNBI ions could be as influential as alpha particles for sawtooth stabilisation in ITER.

The article is organised as follows: models for the sawtooth trigger and plasma evolution are overviewed in Section 2; section 3 presents recent important progress on sawtooth control using localised current drive; in section 4 the effect of counter-NBI on sawteeth in JET is presented; section 5 gives a brief overview of our understanding of sawtooth control using NNBI; the last section contains concluding remarks on the work presented here.

\section{MODELLING THE SAWTOOTH INSTABILITY}

\subsection{THE TRIGGER PROBLEM}

The sawtooth trigger problem is addressed by seeking to correlate equilibrium properties at the onset of the $\mathrm{m}=\mathrm{n}=1$ instability with the crossing of a theoretical stability boundary for the internal kink mode. The theoretical boundary differs as additional physical effects are added into the linearised 'MHD' equations.

It is found that the stability of sawteeth in TCV falls into two broad classes [19] depending on the shape of the plasma cross section. In particular, sawteeth were found to be small for a plasmas 
with large elongation and/or small triangularity. We shall see that such plasma shaping strongly destabilises MHD modes in the core plasma; this includes the internal kink mode, the ideal interchange mode and the resistive interchange mode. Since additional heating shortens the sawtooth period even further, it follows [19] that the sawtooth period for such equilibria could be triggered by the criterion for ideal instability:

$$
\pi \frac{\delta \mathrm{W}}{\mathrm{S}_{1}}<-\frac{\omega_{* \mathrm{i}} \tau_{\mathrm{A}}}{\tau_{\mathrm{n}}}
$$

with $\omega_{* \mathrm{i}}$ the ion diamagnetic frequency and $\mathrm{s}_{1}$ the magnetic shear $\mathrm{s}=(\mathrm{r} / \mathrm{q}) \delta \mathrm{q} / \delta \mathrm{r}$ evaluated at $\mathrm{q}=1$ (denoted by subscript ' 1 '). Here the ideal MHD growth rate $\gamma_{\mathrm{I}} \tau_{\mathrm{A}}=\pi \delta \widehat{\mathrm{W}} / \mathrm{s}_{1}$, so that $\delta \widehat{\mathrm{W}}=\delta \mathrm{W} /\left(2 \pi^{2}\right.$ $\xi_{0} 2 \varepsilon_{1}{ }^{4} \mathrm{R}_{0} \mathrm{~B}_{0}{ }^{2} / \mu_{0}$ ), which can in general account for MHD contributions $\delta \widehat{W} M H D$, and kinetic contributions $\delta \widehat{\mathrm{Wk}}$ from auxiliary hot ion populations and thermal ions in the banana regime. Increased heating would make the MHD contribution to $\delta \widehat{\mathrm{W}}$ more unstable because toroidal contributions [20] and combined toroidal-shaping contributions [21] are increasingly destabilising for increasing $\beta_{\mathrm{p}}$, the poloidal beta at $\mathrm{q}=1$. The contributions to ideal MHD stability have recently been compiled in Ref. [22]:

$$
\begin{aligned}
& \delta \mathrm{w}_{\mathrm{MHD}}=\Delta \mathrm{q} \varepsilon^{2}{ }_{3}\left[\left(0.3-0.5 \frac{\langle\mathrm{r}\rangle}{\langle\alpha\rangle}\right)^{2}-\beta_{\mathrm{p} 3}^{2}\right]+\varepsilon^{4}\left[6 \beta_{\mathrm{p} 1}^{3}\left(1+\beta_{\mathrm{p} 1}\right)+\frac{\beta_{\mathrm{p} 1}\left(1-14 \beta_{\mathrm{p} 1}\right)}{32}\right]-\frac{3 \Delta \mathrm{q}^{3}}{2} \mathrm{e}^{3} \\
& +\frac{\Delta \mathrm{q}}{4} \delta^{2}+\mathrm{e} \varepsilon^{2}\left\{-\frac{3}{2} \beta_{\mathrm{p} 1}+\frac{\Delta \mathrm{q}}{12}\left(156 \beta_{\mathrm{p} 1}^{2}-3 \beta_{\mathrm{p} 1}-12\right)\right\}+\mathrm{e} \delta \varepsilon\left\{3 \beta_{\mathrm{p} 1}+\frac{\Delta \mathrm{q}}{6}\left(4 \beta_{\mathrm{p} 1}-7\right)\right\}
\end{aligned}
$$

with e $=(\kappa-1)(\kappa+1), \kappa$ the elongation, $\delta$ the triangularity, $\langle$ ri $\rangle=r \kappa^{1 / 2}, \Delta \mathrm{q}=1-\mathrm{q}_{0}$ and all quantities are evaluated at $r_{1}$. Figure 1. plots the sawtooth period over a triangularity scan (ranging from large inward pointing to small outward pointing), together with a similar trend in $\delta \widehat{\mathrm{W}}_{\mathrm{MHD}}$ given above and the same quantity calculated numerically [22].

In contrast to the high $\kappa$, or small positive or negative $\delta$ scenarios mentioned above, for more conventional scenarios with moderate $\delta$ and $\kappa$, the sawtooth period in TCV increased with respect to increasing heating power [19]. Such a dependence on heating is consistent with the sawtooth trigger being described by resistive MHD with two fluid effects. Although the $m=n=1$ instability is always unstable in one-fluid resistive MHD, accounting for two fluid effects in the layer reveals stable regions of parameter space which could account for quiescence during sawteeth [23]. The instability criterion can be written in the form $s_{1}>s_{c}(\beta)$. In Ref. [5] it is pointed out that the effects in the layer, which are described by the latter critical shear criterion, are only important when the macroscopic drive $\delta \widehat{\mathrm{W}}$ of the internal kink mode is not strongly stabilising, i.e. there is not a very large energy sink. The criterion for instability is thus given by [5]: 
and

$$
\begin{gathered}
\pi \frac{\hat{\delta \mathrm{W}}}{\mathrm{s}_{1}}<\mathrm{c}_{\rho} \rho_{\mathrm{i}} \\
\mathrm{s}_{1}>\mathrm{s}_{\mathrm{c}}(\beta)
\end{gathered}
$$

where $\rho_{\mathrm{i}}$ is the ion Larmor radius normalised to the $\mathrm{q}=1$ radius, $\mathrm{c}_{\rho}$ a numerical constant of order unity, and $s_{c}(\beta)$ is a critical shear, governed essentially by the pressure profile $[5,23,24,25]$, and also contains a numerical constant $c_{r}$ of order unity. The definition of $s_{c}(\beta)$ depends on the regime of interest. It is for example defined for instability in the ion kinetic regime, or resistive regime, by Eqs.5(b) and 5(a) of [12] respectively.

\subsection{PLASMA EVOLUTION DURING RAMP PHASE}

In order to interpret the sawtooth period in present day experiments, and predict the sawtooth period in planned regimes and future experiments, it is clear that the transport between sawteeth (the ramp phase) must be modelled accurately. An estimation of the current diffusion can be obtained upon considering the leading order tokamak expressions for the induction equation $\delta \mathrm{B}_{\theta} / \delta \mathrm{t}=\mathrm{E}_{\phi}^{\prime}$, Ohm's law $\mathrm{E}_{\phi} \approx \eta \mathrm{J}_{\mathrm{Ohm}}$ and Amperes law $\mu_{0}\left(\mathrm{j}_{\mathrm{Ohm}}+\mathrm{j}_{\mathrm{cd}}+\mathrm{j}_{\text {boot }}\right)=\left(\mathrm{rB}_{\theta}\right)^{\prime}$, where $\mathrm{x}^{\prime} \equiv \delta \mathrm{x} / \delta \mathrm{r}$, and $\mathrm{j}_{\mathrm{Ohm}}+\mathrm{j}_{\mathrm{cd}}+$ $\mathrm{j}_{\text {boot }}$ is the total current, comprising the Ohmic current, driven current and bootstrap current. These equations can be written as an evolution equation in the poloidal magnetic field: $\delta B_{\theta} / \delta \mathrm{t}=\left[(\eta / \mathrm{r})\left(\mathrm{rB} \theta_{\theta} / \mu_{0}\right)^{\prime}-\eta\left(\mathrm{j}_{\mathrm{cd}}+\mathrm{j}_{\text {boot }}\right)\right]^{\prime}$, where $\eta$ is the resistivity, which scales with electron temperature as $\sim \mathrm{T}_{\mathrm{e}}{ }^{-3 / 2}$. Now, for small shear, and using $\mathrm{q} \approx \mathrm{rB}_{0} / \mathrm{R}_{0} \mathrm{~B}_{\theta}$ one obtains:

$$
\frac{\partial \mathrm{q}}{\partial \mathrm{t}}=-\frac{\mathrm{q}}{\mathrm{r}}\left[\frac{2}{\mu_{0}} \frac{\partial \eta}{\partial \mathrm{r}}-\frac{\mathrm{qR}_{0}}{\mathrm{~B}_{0}} \frac{\partial}{\partial \mathrm{r}}\left(\eta \mathrm{j}_{\mathrm{cd}}+\eta \mathrm{j}_{\text {boot }}\right)\right]
$$

and assuming that $(\eta \mathrm{j})^{\prime}>>\mathrm{r}(\eta \mathrm{j})^{\prime \prime}$ :

$$
\frac{\partial \mathrm{s}}{\partial \mathrm{t}}=\frac{2}{\mu_{0} \mathrm{r}} \frac{\partial \eta}{\partial \mathrm{r}}-\frac{1}{\beta_{\theta}} \frac{\partial}{\partial \mathrm{r}}\left(\eta \mathrm{j}_{\mathrm{cd}}+\eta \mathrm{j}_{\mathrm{boot}}\right)
$$

Hence it is seen that variation of the deposition of current drive and/or the conductivity profile modifies the time it takes to reach the critical shear criterion of Eq.3, and hence presumably the sawtooth period.

The effect on sawteeth of modifying the conductivity profile is demonstrated in Fig.2. It is clear that the predictions of the effects of differing ECH sources are qualitatively and essentially quantitatively validated by these TCV discharges [12]. From Eq.5 it is seen that heating outside the inversion radius, such that $\eta^{\prime}$ is reduced at $r_{1}$, reduces $\delta s / \delta$ t in turn (here inversion radius refers to the radius at which the temperature is unchanged over the crash event). Thus the data in Fig. 2 commences with long 
sawteeth. Another beam is deposited close to the magnetic axis, and is then swept through the inversion radius. Again, from Eq.5, the effect on the local conductivity profile initially gives rise to an increase $\delta \mathrm{s} / \delta \mathrm{t}$, and as the beam crosses to the outside of the inversion radius, $\delta \mathrm{s} / \delta \mathrm{t}$ is strongly reduced. In the PRETOR-ST simulations [12], the variation in $\delta s / \delta$ t modifies the sawtooth period because of the differing timescales over which the critical shear criterion of Eq. 3 is reached. It should be noted that Eq. 2 is always satisfied for these experiments which are devoid of energetic minority ions.

It is recognised that simulations of the type shown in Fig.2 assume Kadomsev [26] full reconnection at the crash. Consequently the transport during the ramping phase commences with $\mathrm{s}=0$ and $\mathrm{r}_{1}=0$. Clearly, if full reconnection does not actually occur, transport during the ramp based on Kadomsev initial conditions would over-estimate the sawtooth period if other 'free' parameters (e.g. in $\left.s_{c}(\beta)\right)$ cannot be employed to artificially hasten the triggering event. A relaxation process which does not give rise to such a small shear at the end of the crash phase (and beginning of the next ramp) is based on Taylor relaxation [27]. Evaluation of the region of instability of the resistive interchange mode in MAST [28] indicates that this secondary instability, which could be responsible for the triggering of the Taylor relaxation, is unstable beyond the $q=1$ surface for elongated plasmas. A convincing proof of this trigger mechanism would be the observation of precursorless sawteeth in strongly elongated discharges, and sawteeth with precursors in more conventional (moderate triangularity and elongation) discharges.

Pellet injection into the core quite frequently results in the creation of a high density closed tube of plasma on the $q=1$ surface [29]. Observations [28] of these so called snakes surviving consecutive crash events in the MAST experiments mentioned above indicate that the $\mathrm{q}=1$ radius undergoes only a small variation over the sawtooth cycle. Nevertheless it should be noted that a high degree of temporal resolution would be required in order for such observations to imply that only a small amount of reconnection occurs. This follows because, immediately following Kadomsev reconnection, the rate of change of the $\mathrm{q}=1$ radius is initially very fast, due to its dependence on the vanishingly small magnetic shear:

\section{CONTROL OF SAWTEETH BY CURRENT DRIVE}

Experiments in TCV [10], and more recently in ASDEX-Upgrade [13], demonstrate that the addition of small amounts (less than a few percent of the plasma current) of co-ECCD to ECH in the sweep in the resonance location enhances the trend observed in Fig.2. This can be understood upon inspection of Eq.5 where it is seen that cocurrent drive with resonance location outside $\mathrm{q}=1$, thus giving rise to $\delta \mathrm{j}_{\mathrm{cd}} \delta \mathrm{r}_{\mathrm{r}_{1}}>0$, slows down the evolution of s. Clearly, co-ECCD inside $\mathrm{q}=1$ reduces the sawtooth period. The variation of the plasma evolution and consequent sawtooth period is clearly demonstrated in simulations [12] which account for the combined effects of localised heating and current drive.

Perturbations in the current can also be obtained effectively with ICRH upon employing a particular choice of current phasing in the RF antennas [30]. Figure 3 (a) shows recent results from JET [14] demonstrating the crucial differences between $-90^{\circ},+90^{\circ}$ and dipole phasings, over a 
magnetic field and plasma current scan in discharges employing first harmonic heating of minority hydrogen in a deuterium $\mathrm{D}(\mathrm{H})$ plasma on the high field side (HFS). A large region encompassing both sides of the inversion radius is distinguished within which the resonant surface can be localised in order to obtain large sawteeth for the case of $+90^{\circ}$ and vanishingly small sawteeth for the $-90^{\circ}$ case. Again these results are understood in terms of a change in the sign and magnitude of $\delta \mathrm{j}_{\mathrm{cd}} / \delta \mathrm{r}$.

Unlike co-ECCD with resonant position inside $\mathrm{q}=1$, one does not see significant destabilisation of sawteeth for the $+90^{\circ}$ case as the resonant surface moves inside the inversion radius. As pointed out in Ref. [31] there are two competing mechanisms at work, namely the effect of the minority ions on the current perturbation, and also the stabilising effect of hot ' $h$ ' trapped minority ions on the macroscopic potential energy

$$
\delta \hat{\mathrm{W}}_{\mathrm{kh}}=\frac{1}{\pi\left(\varepsilon_{1}\right)^{1 / 2}} \quad \beta_{\mathrm{ph}} \text { with } \beta_{\mathrm{ph}}=-\frac{2 \mu_{0}}{\mathrm{~B}_{0}} \quad{ }_{0}^{\mathrm{r} 1} \mathrm{dr}\left(\frac{\mathrm{r}}{\mathrm{r}_{1}}\right)^{3 / 2} \frac{\mathrm{dP}_{\mathrm{h}}}{\mathrm{dr}}
$$

This latter stabilising effect is most significant for ions heated with the $+90^{\circ}$ phasing because these particles are pinched inwards toward the magnetic axis [32], thus creating larger fast ion pressure gradients within the $\mathrm{q}=1$ surface. Intuitively, the opposite occurs for the $-90^{\circ}$ phasing. Nevertheless, all three phasing conditions give rise to kinetic stabilisation of the sawteeth as the resonant surface approaches the magnetic axis.

Crucial recent experiments in JET [15] demonstrate that creating current perturbations with $\delta \mathrm{j}_{\mathrm{cd}} / \delta \mathrm{r}<0$ can control (destabilise) sawteeth even where the plasma additionally comprises a fast ion population in the core yielding a large stabilising macroscopic drive of the form of Eq.6. It is noted in Ref. [15] that despite this stabilising contribution, increasing the magnetic shear can shorten the sawtooth period via the shear dependence in the macroscopic threshold for instability of the internal kink mode in the ion kinetic regime, i.e. Eq.2. Shown in Fig.3(b) are long sawteeth initially stabilised by using central hydrogen minority heating and $+90^{\circ}$ phasing. At 19 seconds the same species is also heated close to the inversion radius on the HFS with $-90^{\circ}$ phasing. As the discharge evolves, and the inversion radius converges with the hydrogen resonance position of the $-90^{\circ}$ phasing, the sawteeth become very short.

Clearly in ITER it may be necessary to use techniques to modify $s_{1}(t)$ in order to control sawteeth lengthened by the predicted large energy sink from the kinetic contribution of trapped alpha particles in the core. Indeed, evidence of seeding of NTMs through sawteeth lengthened by a He4 population heated into the MeV range with third harmonic ICRH [33], confirms that this is likely to be crucial. The use of ITER relevant second harmonic ICCD [6] has been successful in controlling sawteeth and avoiding the triggering of NTMs with N close to the ideal MHD limit.

\section{COUNTER NBI AND TOROIDAL ROTATION}

In this section the influence of plasma rotation on sawteeth is considered. Figure 4 shows two otherwise similar recent JET discharges [16] which have opposite NBI orientation (relative to the 
direction of the toroidal plasma current and toroidal magnetic field). It is seen that the co-NBI discharge has increasingly large sawteeth for increasing heating power up to a maximum of $\mathrm{P}_{\mathrm{NBI}} \approx 3.8 \mathrm{MW}$, while the cntr-NBI discharge has sawteeth with quiescent periods close to the Ohmic timescale throughout the ramp up and ramp down in the neutral beam power.

A clear difference between the two discharges shown in Fig. 4 is the change in sign in the plasma rotation relative to the toroidal current and magnetic field. Figure 5(a) and (b) plots the measured differential toroidal plasma rotation $\Delta \Omega=-\left.\mathrm{r} \Omega^{\prime}\right|_{\mathrm{r}_{1}}$ and the ion diamagnetic frequency $\omega_{\mathrm{pi}}^{*}\left(\mathrm{r}_{1}\right)$ with respect to time for the shots shown in Fig.5. Here $\Omega^{\prime}=\delta \Omega / \mathrm{dr}$, and $\omega_{\mathrm{pi}}^{*}=-\mathrm{qP}_{\mathrm{i}}^{\prime} /\left(\mathrm{eZn}_{\mathrm{i}} \mathrm{B}_{0} \mathrm{r}\right)$. It is seen that for co-injection $\Delta \Omega \approx 2 \omega_{\mathrm{pi}}{ }$, while for the counter-injection discharge $\Delta \Omega \approx-2 \omega_{\mathrm{pi}}^{*}$. This scaling of the differential plasma rotation relative to the ion diamagnetic frequency is required in order to assess the stabilising contribution of effectively collisionless trapped ions. The response of the NBI hot ' $h$ ' ions to the internal kink mode is essentially given by Eq.(6) for such relatively small sheared flow [34] for which $\widehat{\Delta} \Omega<<\left\{\left\langle\omega_{\mathrm{mdh}}\right\rangle, \omega_{* \mathrm{~h}}\right\}$, where $\left\langle\omega_{\mathrm{mdh}}\right\rangle$ is the magnetic drift and $\omega_{* \mathrm{~h}}$ the diamagnetic frequency of the hot ions. In contrast, the collisionless response of the thermal ions ' $\mathrm{i}$ ' is significantly modified by sheared flow [35] because $\Delta \Omega \sim\left\{\left\langle\omega_{\text {mdi }}\right\rangle, \omega_{* \mathrm{i}}\right\}$. Figure 5 (c) plots the kinetic contribution from thermal ions $\delta \mathrm{W}_{\mathrm{ki}}$ as a function of $\Delta \Omega / \omega_{* \mathrm{pi}}$ for typical core plasma profiles and for differing $\eta_{\mathrm{i}}=\mathrm{d} \ln \mathrm{T}_{\mathrm{i}} / \mathrm{d} \ln \mathrm{n}_{\mathrm{i}}$. Variation in $\Delta \Omega$ was undertaken by changing the central plasma rotation $\Omega_{0}$ for a fixed profile $\Omega / \Omega_{0}=1-(\mathrm{r} / 2 \mathrm{a})^{2}$ as in Ref. [35]. Indicated in Fig.5(c) are the approximate relevant normalised differential frequencies for the co and cntr-NBI discharges depicted in Figs.4(a) and (b). This serves to illustrate the influence of sheared flow on stability for the discharges of concern here.

Figure 4 also plots the terms involved in the criterion of Eq. 2 for the co-NBI Pulse No: 60768 and reverse B Pulse No: 59705. This is achieved by computing the time evolution of the quantities involved by taking data directly from the available JET diagnostics and post-processing codes such as PENCIL [36]. For the co-NBI case the large increase in the sawtooth period coincides with the approximate time during the ramp when the macroscopic instability criterion of Eq.2 is no longer satisfied [24]. The sawtooth period essentially returns to an Ohmic timescale $\tau_{\text {saw }} \sim 80 \mathrm{~ms}$ at the point during the ramp down phase when Eq. 2 is satisfied. Both $\delta \mathrm{W}_{\mathrm{ki}}$ and $\delta \mathrm{W}_{\mathrm{kh}}$ are responsible for the stabilisation during the ramp. In contrast, for the cntr-NBI case, the sawtooth period does not rise above that of Ohmic sawteeth, and it is found that the thermal ion kinetic contribution $\delta \mathrm{W}_{\mathrm{ki}}$ is negligible, and the hot ion kinetic term $\delta \mathrm{W}_{\mathrm{kh}}$ is also smaller than the corresponding term for the forward B Pulse No: 60768. Since the instability criterion Eq.2 is always met, the trigger occurs when the criterion $s_{1}>s_{c}(\beta)$ is satisfied. Since the sawtooth period is similar to that of Ohmic plasmas, it is reasonable to suppose that the energetic particles do not significantly modify the current penetration time. Furthermore, the counter current drive is small, and measurements of the inversion radius cannot discern an effect on the q profile.

Figure 6 demonstrates that it is necessary to have at least 7MW of cntr- NBI power before kinetically stabilised sawteeth are observed. This contrasts with only requiring $2 \mathrm{MW}$ of co-NBI. For discharges where the deposition of heating is concentrated deeply in the centre (e.g. with normal 
injection), the sawtooth period is much smaller than Ohmic sawteeth. For moderate cntr-NBI power the sawteeth a very regular, while for large cntr-NBI power or moderate co-NBI power the sawtooth period varies sigificantly (see error bars in Fig.6) over the two second stationary interval during which measurements are made. A clear broad minimum in the sawtooth period is observed with a timescale less than half that of Ohmic sawteeth. The physical reason for this is as yet uncertain, but in contrast with the speculation in Ref. [11], it would appear that the relatively small and broadly deposited current drive is insignificant in all the discharges assessed here. It is possible however that the deep penetration of NBI ions could modify the conductivity profile in a similar way to that of on-axis ECH (see Sec. 2.2 and Fig.2). The sensitive influence of the conductivity profile on sawteeth is made possible because, unlike co-NBI where the sawtooth period is determined by the criterion for macroscopic instability [24] of Eq.2, for cntr-NBI of moderate heating power the criteria of Eq. 2 is always met. Hence the trigger is determined by the critical shear criterion of Eq.3, which, as we have seen, is particularly sensitive to changes in the local conductivity. Finally, it is noted that there are other ways in which toroidal plasma rotation can modify stability. A similar variation of the sawtooth period with heating power and injection orientation has emerged in TEXTOR [37] and MAST [38]. However, in both TEXTOR and MAST the minimum in the sawtooth period corresponds closely to a vanishing precursor frequency, while with e.g. 4MW of cntr-NBI in JET the MHD mode frequency is the same order of magnitude as the toroidal plasma rotation frequency. Furthermore, in MAST, the toroidal rotation frequency is an order of magnitude larger than JET discharges with the same NBI power, and hence it is quite possible that centrifugal effects could be important [39]. In addition, if $\Delta \Omega \sim\left\{\omega_{\text {mdh }}, \omega_{* \mathrm{~h}}\right\}$, the kinetic response of the NBI minority ions themselves could be modified significantly by sheared flow [34]. Finally, other effects which have not been considered here include the possibility that changes in the bulk plasma momentum by NBI could modify the toroidal coupling of perturbations on the $1 / 1$ and $2 / 1$ surfaces [40].

\section{NEGATIVE ION BASED NEUTRAL BEAM INJECTION}

Experiments in JT-60U using 350keV negative ion based neutral beam injection (NNBI) in the direction of the plasma current have yielded long sawteeth [17]. The dependence of the sawtooth period on electron temperature is found to be in excess of the scaling $\sim \mathrm{T}_{\mathrm{e}}^{3 / 2}$ due solely to resistive diffusion. However, these long sawteeth cannot be explained simply in terms of the usual kinetic stabilisation observed for conventional NBI or RF minority ions because, for the strongly tangential injection employed, the fraction of trapped ions is presumably small. Nevertheless, the tangential injection is unbalanced, and it is found that the parallel velocity asymmetry in the distribution function gives rise to an important kinetic contribution when finite orbit effects are taken into account [18]. It is found that on-axis co- NNBI and strongly off-axis cntr-NNBI stabilise the internal kink mode, while on-axis cntr-NNBI and strongly off-axis co-NNBI are destabilising. Here strongly off-axis refers to the possibility of obtaining positive fast ion pressure gradients at $q=1$. In ITER, tangential $1 \mathrm{MeV}$ NNBI is being planned. It is found that [41] the stabilising contribution from this 
population of NNBI ions in ITER could be as large as the stabilising contribution from trapped alpha particles if:

$$
\left.\mathrm{r} \frac{\mathrm{dP}_{\mathrm{h}}}{\mathrm{dr}}\right|_{\mathrm{r}_{1}}-\frac{1}{\varepsilon_{1}^{1 / 2}},{ }_{0}^{\mathrm{r} 1} \mathrm{dr}\left(\frac{\mathrm{r}}{\mathrm{r}_{1}}\right)^{3 / 2} \frac{\mathrm{dP}_{\alpha}}{\mathrm{dr}}
$$

where $\mathrm{P}_{\mathrm{h}}$ and $\mathrm{P}_{\alpha}$ respectively refer to the pressure from unbalanced circulating NNBI hot ions and alpha particles. Such a scaling could be satisfied for tangential injection of NNBI ions where $\mathrm{dP}_{\mathrm{h}} / \mathrm{dr}$ is peaked off-axis, i.e. close to $q=1$. For NNBI power of $35 \mathrm{MW}$ and alpha heating power of 80 MW [42] it is quite possible that one finds that $\mathrm{dP}_{\mathrm{h}} / \mathrm{dr}>\mathrm{dP}_{\alpha} / \mathrm{dr}$ close to $\mathrm{q}=1$.

The effect of the driven current from NNBI is now briefly addressed. TRANSP simulations [42] of standard ELMy H-mode scenarios in ITER demonstrate that the NNBI population does significantly modify (broaden) the q-profile. Depending on the injection geometry employed, cocurrent drive with a non-monotonic profile could readily be created [43]. For such a non-monotonic NNBI current drive profile $j_{\text {NNBI }}(r)$, the safety factor in the core could increase with respect to time. This can be understood from Eq. 4 for which $\delta\left(\eta \mathrm{j}_{\mathrm{cd}}\right) / \delta \mathrm{r}>0$ yields $\delta \mathrm{q} / \delta \mathrm{t}>0$ providing that the effect of current drive dominates over conventional resistive diffusion in the core. The current drive efficiency should at the very least be sufficient to significantly delay the first creation of a $q=1$ surface following start up. This application of current drive could in principle therefore be employed in order to avoid or delay sawteeth rather than provide sawtooth control by means of destabilisation.

\section{CONCLUSIONS}

This article presents an overview of recent advances in the field of sawtooth control. It includes progress on sawtooth destabilisation using both localised electron and ion based heating and current drive. Consistent simulations of TCV and JET discharges demonstrate that sawtooth destabilisation occurs as a result of enhancement to the current diffusion close to the $q=1$ rational surface [12]. The ability to control sawteeth, initially stabilised by an energetic trapped ion population, is a particularly important step forward given the expectation that alpha particles will lengthen sawtooth periods in ITER. Recent experiments in JET with counter-NBI highlights the influence of plasma rotation in present day tokamaks. While the expected small momentum induced flow is not predicted to strongly influence sawteeth in ITER, the effect of unbalanced NNBI is predicted to strongly stabilise sawteeth through kinetic stabilisation of the internal kink mode, and possibly by slowing down resistive diffusion in the core.

\section{ACKNOWLEDGEMENT}

This work was partly funded by the Fonds National Suisse de la Recherche Scientifique. 


\section{REFERENCES}

[1]. ITER Physics Basis Editors et al., Nucl. Fusion 39, 2137, (1999).

[2]. D.J. Campbell, D.F.H. Start, J.A. Wesson, D.V. Bartlett, et al., Phys. Rev. Lett. 602148 (1988).

[3]. C.K. Phillips J. Hosea, E. Marmar, M.W. Phillips, et al., Phys. Fluids B 4, 2155 (1992).

[4]. J.P. Graves, K.I. Hopcraft, R.O. Dendy, R.J. Hastie, et al., Phys. Rev. Lett. 84, 1204 (2000).

[5]. F. Porcelli, D. Boucher and M.N. Rosenbluth, Plasma Phys. Controlled Fusion 38, 2163 (1996).

[6]. O. Sauter, E. Westerhof, M.L. Mayoral, B. Alper, et al. Phys. Rev. Lett. 88, 105001 (2002).

[7]. D.J. Campbell et al., Proc. 15th Eur. Conf. on Controlled Fusion and Plasma Heating (EPS, Dubrovnik, 1988), Vol. 12B, Part 1, p377.

[8]. F. Porcelli et al., Nucl. Fusion 44, 362 (2004).

[9]. G.A. Bobrovskii et al., Sov. J. Plasma Phys. 13, 665 (1987).

[10]. T.P. Goodman et al. 1999 Proc. 26th EPS Conf. on Control. Fusion and Plasma Physics (Maastricht, 1999) vol 23J (ECA) p1101; Henderson M.A. et al. 2001 Fus. Eng. Des. 53241.

[11]. A.W. Edwards et al., Proc. 19th EPS Conf., Vol. 16C, Part I, p379 (1992).

[12]. C. Angioni, T.P. Goodman, M.A. Henderson and O. Sauter, Nuclear Fusion 43, 455 (2003).

[13]. A. Mück et al. accepted in Plasma Phys. Control. Fusion (2005).

[14]. M.-L. Mayoral, Phys. Plasmas 11, 2607 (2004).

[15]. L.-G. Eriksson, et al., Phys. Rev. Lett. 92, 235004 (2004).

[16]. M.F.F. Nave et al., Proc. 31st EPS Conf. Plasma Physics, London, United Kingdom, June 28 July 2, 2004, P1-162, European Physical Society (2004).

[17]. G.J. Kramer et al., Nucl. Fusion 40, 1383 (2000).

[18]. J.P. Graves, Phys. Rev. Lett. 92, 185003 (2004).

[19]. H. Reimerdes et al., Plasma Phys. Control. Fusion 42, 629 (2000).

[20]. M.N. Bussac, R. Pellat, D. Edery and J.L. Soul'e, Phys. Rev. Lett. 35, 1638 (1975).

[21]. H.G. Eriksson and C. Wahlberg, Phys. Plasmas 9, 1606 (2002).

[22]. An. Martynov, J. Graves, O Sauter, sub Plasma. Phys. Control. Fusion (2005)

[23]. S. Migliuolo, F. Pegoraro and F. Porcelli, Phys. Fluids B 3, 1338 (1991); L. Zakharov, B. Rogers and S. Migliuolo, Phys. Fluids B 5, 2498 (1993).

[24]. C. Angioni, A. Pochelon, N.N. Gorelenkov, K.G. McClements, et al., Plasma Phys. Control. Fusion 44, 205 (2002).

[25]. F.M. Levinton, L. Zackharov, S.H. Batha, J. Manickam and M.C. Zarnstorff, Phys. Rev. Lett. 72, 2895 (1994).

[26]. B.B. Kadomsev, Sov. J. Plasma Phys. 1, 555 (1977).

[27]. C.G. Gimblett and R. J. Hastie, 36, 1439 (1994).

[28]. R.J. Buttery et al., Nuclear Fusion 44, 1027 (2004).

[29]. A. Weller et al., Phys. Rev. Lett. 59, 2303 (1987).

[30]. V.P. Bhatnagar et al., Nucl. Fusion 34, 1579 (1994). 
[31]. F. Porcelli et al., Proc. EPS Conf., Vol. 16C, Part II, p901 (1993).

[32]. L.-G. Eriksson, et al. Phys. Rev. Lett. 81, 1231 (1998)

[33]. M. Mantsinen, et al., Phys. Rev. Lett. 88, 105002 (2002).

[34]. J.P. Graves, et al., Phys. Plasmas 10, 1034 (2003).

[35]. J.P. Graves, R. J. Hastie and K. I. Hopcraft, Plasma Phys. Control. Fusion 42, 1049 (2000).

[36]. D. Bailey Report JET-R 04 (1998).

[37]. H.R. Koslowski, Fusion Sci. Technology 47, 260 (2005).

[38]. R.J. Akers, I.T. Chapman et al., proceedings of EPS 2005

[39]. C. Wahlberg and A. Bondeson, Phys. Plasmas 7, 923 (2000); R. G. Kleva and P. N. Guzdar, Phys. Plasmas 9, 3013 (2002).

[40]. R. Fitzpatrick, C.G. Gimblett and R.J. Hastie, 20th EPS Conf. on Plasma Physics and Contolled Fusion Fusion (Lisbon) vol 17C, part IV, p1335 (1993).

[41]. J.P. Graves, submitted to Phys. Plasmas 2005.

[42]. R.V. Budny, Nucl. Fusion 42, 1383 (2002).

[43]. K. Tobita et. al., Plasma Phys. Control. Fusion 46, S95 (2004). 

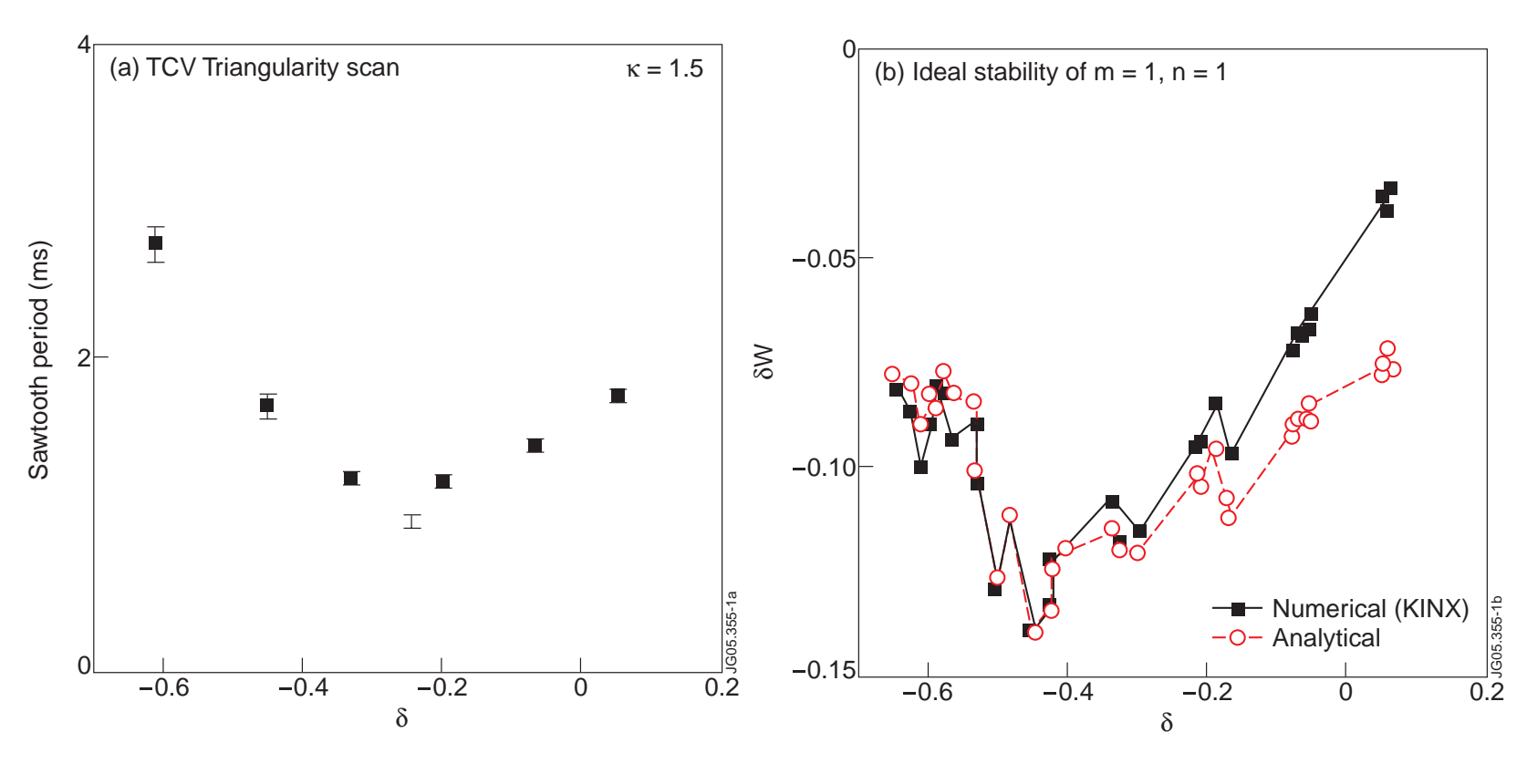

Figure 1: Plot of (a) the sawtooth period in TCV over large inward pointing to small conventional outward pointing triangularity for moderate elongation. The trend is very similar to $(b)$ the ideal stability given by Eq. 1 and with results from the KINX ideal MHD stability code [22].
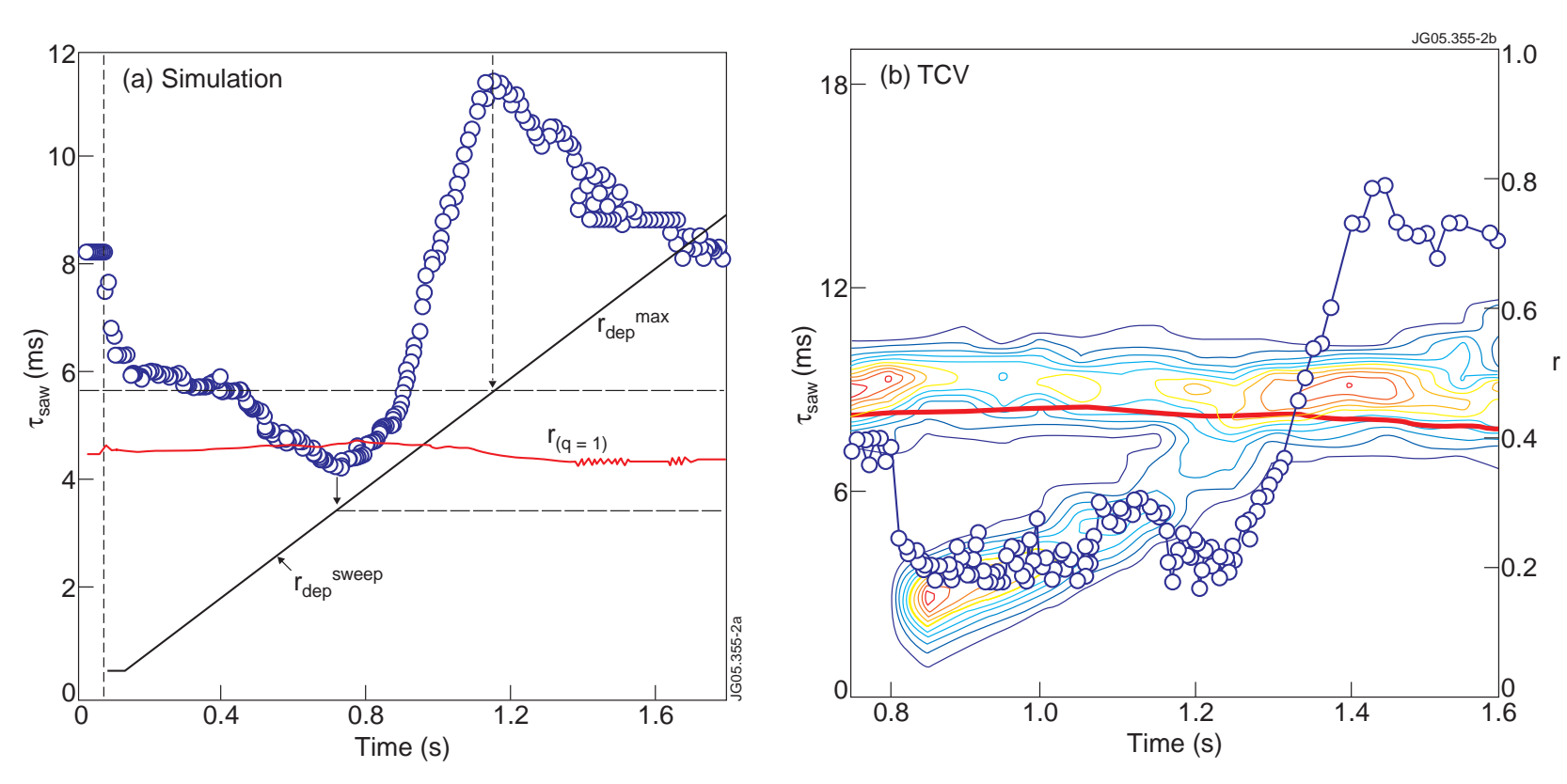

Figure 2: Showing (a) predictions of the sawtooth period using PRETOR-ST and (b) the measured sawtooth period in $T C V$ with beams of ECH orientated just outside $r_{1}$ radius to stabilise the sawteeth, and another beam is then swept through $r_{i n v}$ [12]. Contours in $(b)$ indicate location of heat deposited relative to minor radius $r$. 

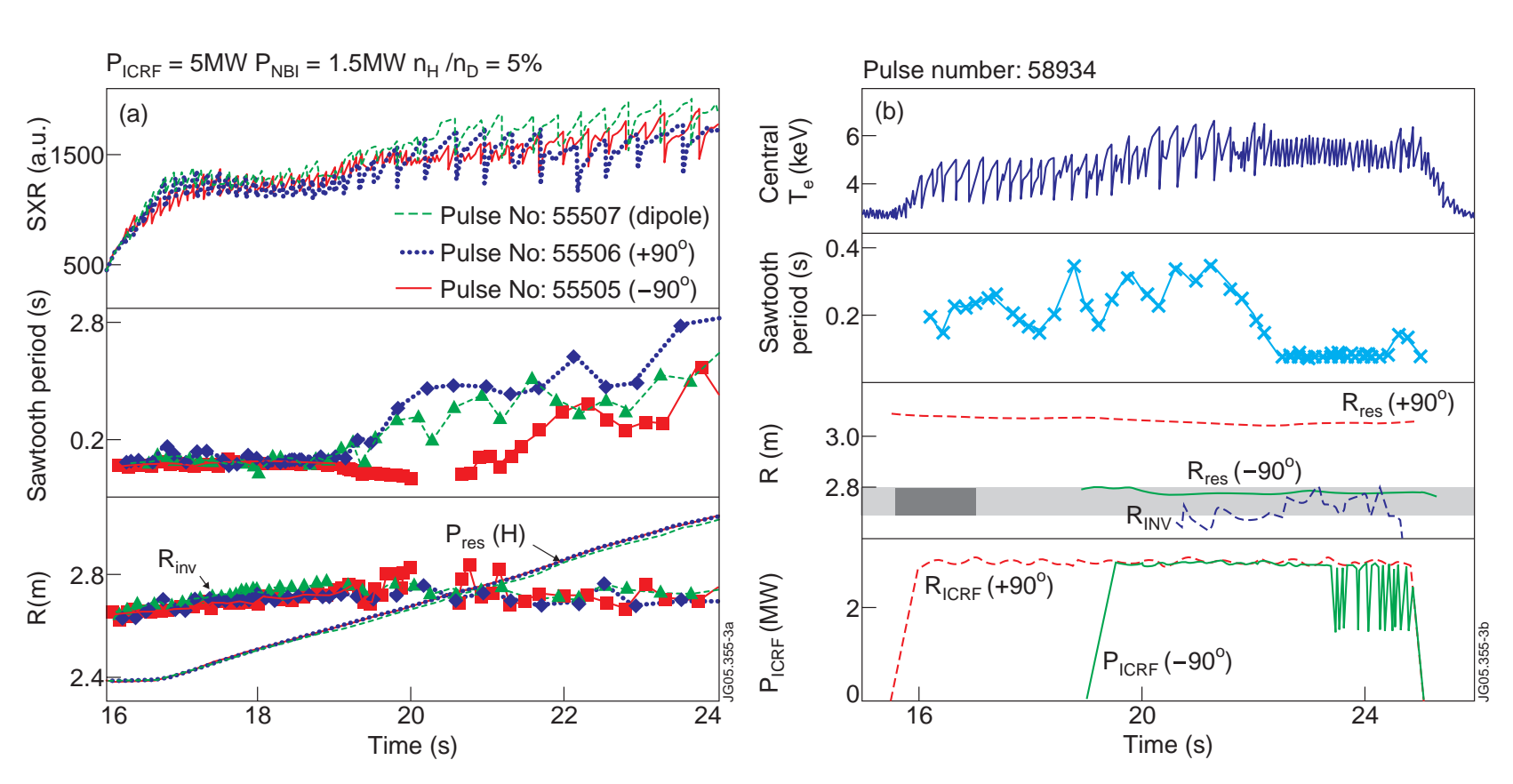

Figure 3:(a): Soft $x$-ray emission, sawtooth period, sawtooth inversion radius $R_{\text {inv }}$ and first harmonic $H$ cyclotron resonance layer $R_{\text {res }}(H)$ for three similar JET discharges [14] for which only the phasing of the ICRF wave was changed: +90 phasing in Pulse No: 55505, -90 phasing in Pulse No: 55506, and dipole phasing in Pulse No: 55507. (b): Pulse No: 58934 in JET [15], plotting the electron temperature, sawtooth period, sawtooth inversion radius $R_{\text {inv }}$ and first harmonic $H$ cyclotron resonance layer $R_{\text {res }}(H)$ for $+90^{\circ}$ and $-90^{\circ}$ phasings, and heating power for the two antennas.
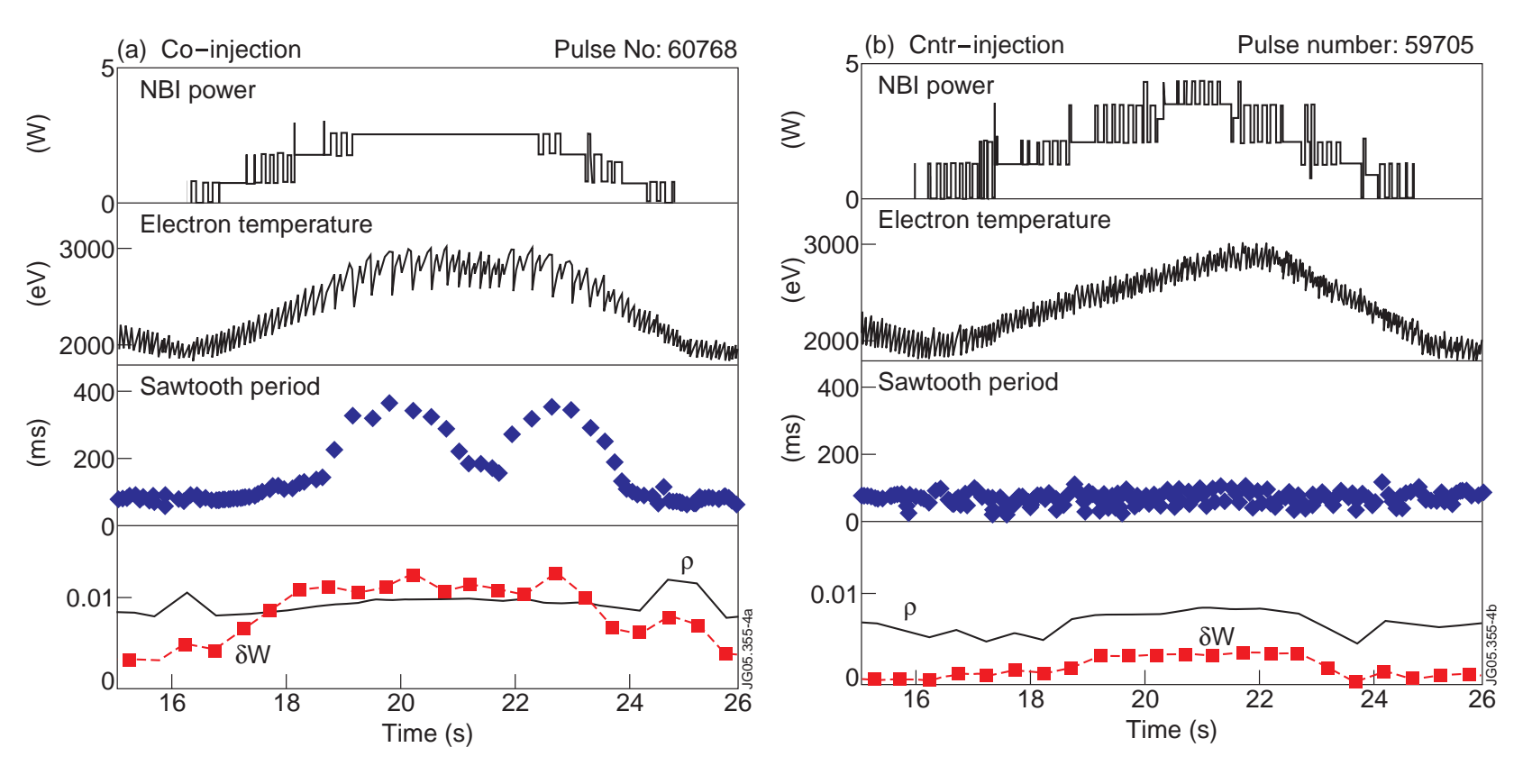

Figure 4: Showing two otherwise similar NBI ramping discharges with opposite NBI orientation [16]. Also compared are the expressions $\delta W=\pi \delta \hat{W} / s_{1}$ and $\rho=c_{r} \rho_{i}$ in the macroscopic threshold instability criterion of Eq.2. 


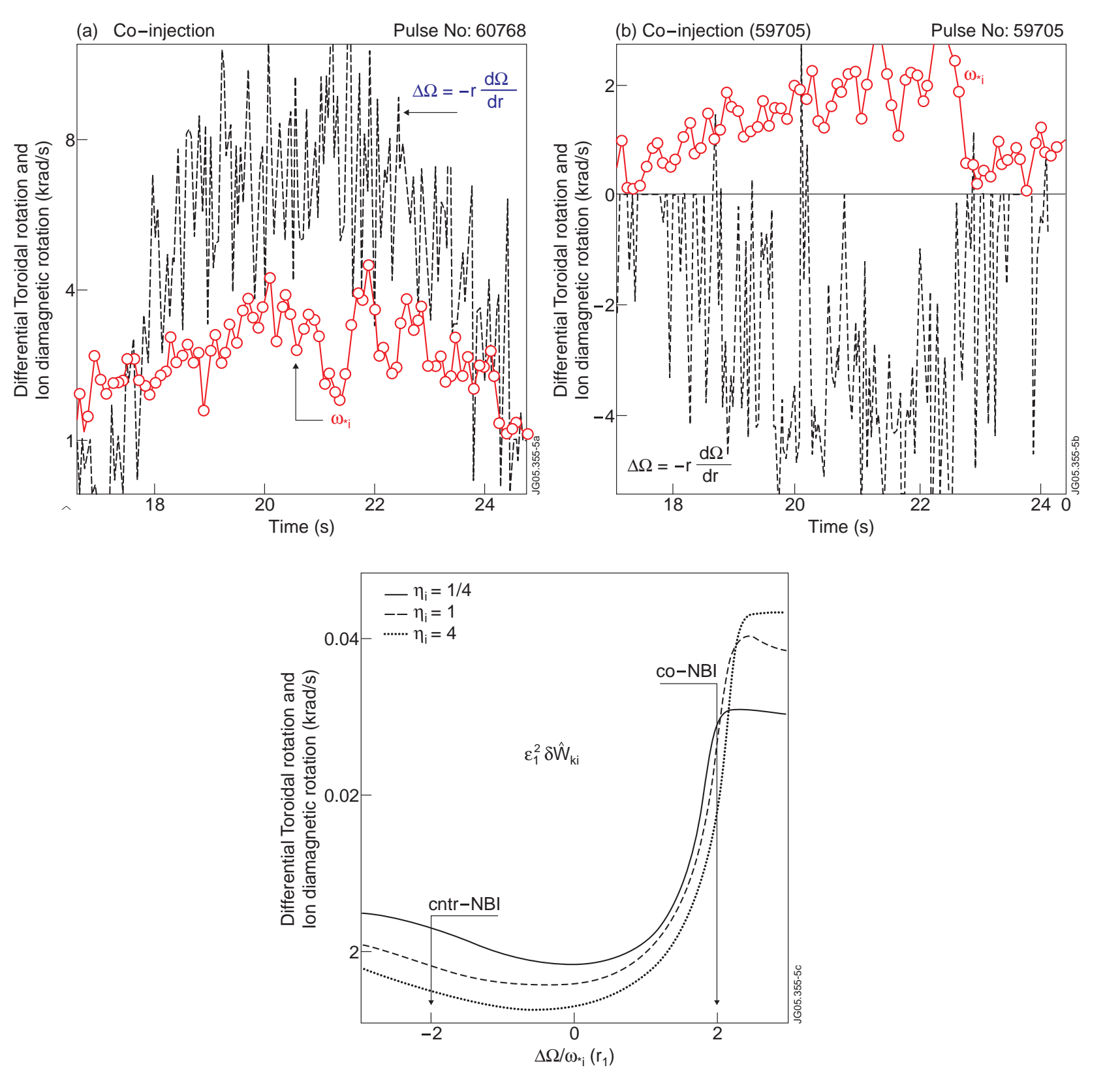

Figure 5: Showing $\Delta \Omega$ and $\omega_{* p i}$ for (a) the co-NBI and (b) the cntr-NBI discharges shown in Fig.4. The variation of $\epsilon^{2}{ }_{1} \delta W_{k i}$ with respect to $\Delta \Omega / \omega_{* p i}$ is shown in (c). 


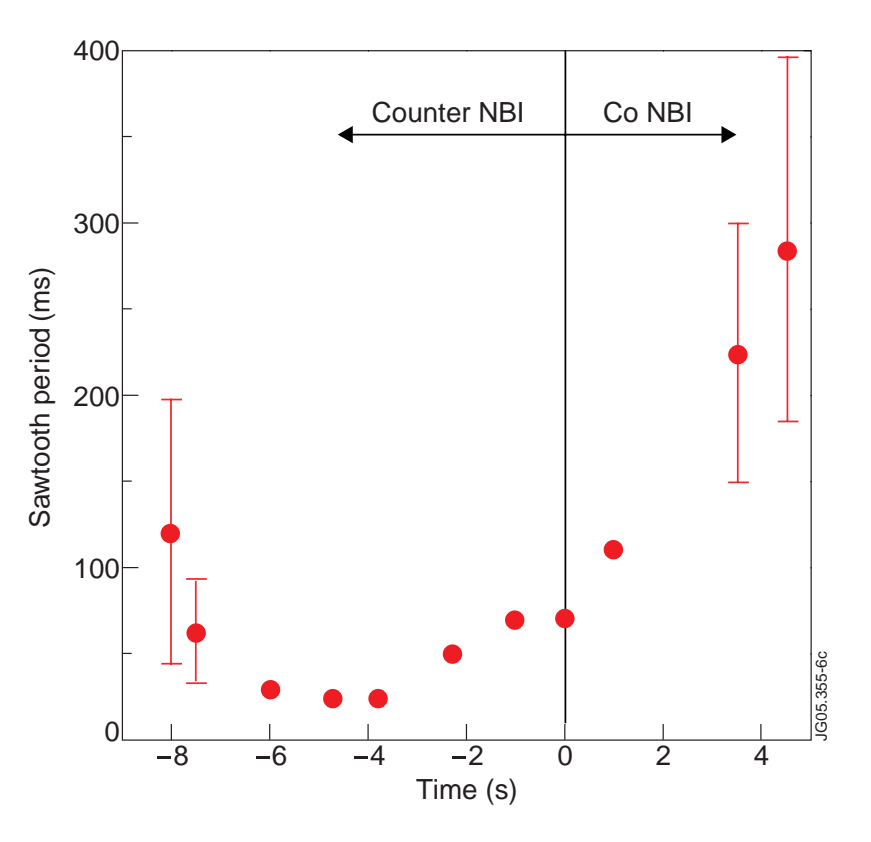

Figure 6: Showing the dependence of the sawtooth period on $N B I$ heating power and direction relative to the plasma current in a series of dedicated JET discharges [16]. Error bars indicate variation of sawtooth period over the measured two second interval during which measurements we taken. 